\title{
SIRColab: Explorando Estruturas para o Estudo Colaborativo de Textos Científicos Remotamente
}

\begin{abstract}
André V. Tardelli ${ }^{1}$, Luciana S. Brito ${ }^{1}$, Pablo E. L. Villanueva ${ }^{1}$, Adriana S. Vivacqua ${ }^{1}$, Juliana B. S. França ${ }^{2}$, Angélica F. S. Dias ${ }^{34}$

${ }^{1}$ Programa de Pós Graduação em Informática - Instituto de Computação - Universidade Federal do Rio de Janeiro (UFRJ) - Rio de Janeiro - RJ - Brasil. ${ }^{2}$ DECOMP/PPGMMC/PPGE - Universidade Federal Rural do Rio de Janeiro (UFRRJ) - Rio de Janeiro - RJ - Brasil. ${ }^{3}$ Instituto Tércio Pacitti de Aplicações e Pesquisas Computacionais (NCE) - Rio de Janeiro - RJ - Brasil. ${ }^{34}$ Programa de Pós Graduação em História das Ciências e das Técnicas e Epistemologia (HCTE) - Universidade Federal do Rio de Janeiro (UFRJ) - Rio de Janeiro - RJ - Brasil

Andretardelli@gmail.com, lbrito@cecierj.edu.br, plopezv@unmsm.edu.pe, avivacqua@dcc.ufrj.br, julibsf@gmail.com, angelica@nce.ufrj.br

Abstract. Higher education students may find it difficult to conduct their research of scientific articles in a quick and collaborative way with the actual technologies. To facilitate the development of academic material for groups in remote environments, a structure is proposed for the summarization of relevant information in Remote Collaborative Environments. Based on the Design Science Research (DSR) methodology, this artifact consists of a form containing the most relevant information to be searched for when reading an article. The effectiveness of this structure investigates the main reading strategies and group combinations based on their personalities.
\end{abstract}

Resumo. Estudantes de ensino superior podem encontrar dificuldades para realizar suas pesquisas de artigos científicos de forma rápida e colaborativa com as tecnologias atuais. Para facilitar o desenvolvimento de material acadêmico para grupos em ambientes remotos, foi proposta a estrutura para a Sumarização de Informações Relevantes em Ambientes Remotos Colaborativos (SIRColab). Fundamentado na metodologia Design Science Research (DSR), este artefato consiste em um formulário contendo as informações mais relevantes a serem buscadas durante a leitura de um artigo. A avaliação investiga as principais estratégias de estudo e combinações de grupos baseados em suas personalidades.

\section{Introdução}

Nos últimos anos, foi notável o aumento de tecnologias e programas de aprendizado à distância, aumentando o alcance de conteúdos espalhados pela internet [D'angelo 2018]. A adoção de novos paradigmas de trabalho e aprendizado virtual implicaram na adoção de diferentes estratégias para a realização de tarefas distintas do cotidiano.

Uma das tarefas mais árduas deste contexto é a realização de atividades de maneira colaborativa. A implementação de um ambiente colaborativo remoto é mais árdua do que o seu análogo presencial, visto que a comunicação se torna menos frequente e algumas atividades podem ser realizadas de maneira assíncrona pelos participantes [Kraut, 2002]. 
A colaboração se torna um aspecto imprescindível especialmente com relação às atividades em grupo que envolvem leitura e interpretação de artigos acadêmicos por estudantes universitários remotamente.

Certas situações podem surgir durante o estudo de um artigo acadêmico de maneira colaborativa, derivadas de aspectos comportamentais e externos entre os participantes. Segundo Shafie (2010), estudantes podem possuir conflitos durante a discussão devido às suas diferentes personalidades e características. Devido aos diferentes processos cognitivos, a qualidade da busca por tópicos relevantes pode acabar sendo comprometida, acarretando a perda do tempo disponibilizado para conceber o trabalho. Outro aspecto que afeta diretamente a qualidade dos trabalhos realizados é o déficit de tempo e de conhecimentos prévios com relação a métodos efetivos de leitura e pesquisa. Shafie (2010) destaca a importância do tempo disponibilizado para realizar uma atividade, e que muitas vezes esse tempo acaba sendo desperdiçado pois os estudantes não possuem uma orientação clara de por onde começar a sua pesquisa.

O problema desta pesquisa é direcionado aos estudantes do ensino superior, que não possuem uma forma otimizada para estruturar as informações de um artigo científico de maneira rápida e colaborativa durante o trabalho remoto. Como proposta para a solução, foi concebida a criação de um artefato apoiado no paradigma Design Science Research (DSR), no modelo de colaboração 3C e no modelo de tipologia de personalidade Myers-Briggs [Myers 1995; Pimentel 2016; Pimentel 2019]. O artefato criado consiste em uma estrutura para a Sumarização de Informações Relevantes em Artigos Remotos de maneira Colaborativa (SIRColab). O objetivo desta investigação é explorar de que forma pode-se auxiliar grupos de pesquisa, gerando direcionamento conciso da leitura e levantamento colaborativo das informações principais de publicações científicas, quando analisadas de forma remota.

O artefato foi avaliado por um estudo de caso realizado remotamente através de uma atividade com um intervalo de tempo delimitado aplicada em uma turma de pósgraduação de uma universidade pública do Rio de Janeiro. O objetivo da avaliação foi verificar se o artefato resolvia o problema de pesquisa e quais mudanças poderiam ser realizadas a fim de melhorar a sua performance, através da análise das estratégias principais utilizadas pelos grupos do artefato em um estudo de caso.

O artigo é dividido em 7 seções. Na seção 2: trabalhos relacionados; na seção 3: concepção do artefato e metodologia de pesquisa utilizada; seção 4: a avaliação do artefato; seção 5: análise dos resultados; seção 6: conclusão e próximos passos.

\section{Trabalhos Relacionados}

Estratégias de leitura: foram identificados os trabalhos de Agger et al. (2017) e Sun (2020). Agger et al. (2017) sugerem que pesquisadores podem se beneficiar ao lerem seções em ordem diferente da pré-definida. Sun (2020) diferencia "leitura ativa", que ocorre ao ler um artigo com questões em mente, de "leitura passiva", que ocorre ao ler sem expectativa ou propósito determinados. $\mathrm{O}$ autor dá sugestões de questionamentos importantes a serem feitos sobre um artigo durante a sua leitura.

Personalidade em grupos de trabalho: foram identificadas publicações de Tardelli et al. (2019), Toseland e Rivas (2005), Van Knippenberg e Schippers (2007) e Tardelli et al. (2020). É possível realizar projetos mais complexos em menor tempo através da atribuição de tarefas, estimulando aspectos sociais e colaborativos de grupos. A atribuição 
de papéis também é um fator importante para garantir a satisfação geral dos integrantes durante a concretização de objetivos [Toseland e Rivas 2005]. Van Knippenberg e Schippers (2007) estabelecem que as análises destas características podem contribuir para a performance de grupos, desde que usadas para otimizar o consenso geral entre participantes e apaziguar conflitos.

Tardelli et al. (2019), investigaram a formação de grupos na aprendizagem colaborativa, fazendo um levantamento de informações de acordo com as características aderentes do seu perfil comportamental baseado na tipologia Myers Briggs. A tipologia organiza os indivíduos em 16 tipos de personalidade, definidos por acrônimos gerados pelos quatro pares de dicotomias que formam seu tipo. As dicotomias são divididas em 4 pares: Introversão (I) e Extroversão (E), que representam a sua fonte de energia; Sensação (S) e Intuição $(\mathrm{N})$, que representam seu modo de perceber o mundo; Pensamento (T) e Sentimento (F), mostrando sua maneira de decidir e lidar com emoções e Julgamento (J) e Percepção (P), mostrando sua abordagem no trabalho, planejamento e tomada de decisões.

Tardelli et al. (2020) propôs a formação de grupos através do Framework FGT, configurando grupos sinérgicos a determinada atividade. As atividades são discernidas em quatro categorias: Pesquisa requer Organização e Planejamento (combinação da praticidade da dicotomia $\mathrm{S}$ e da ordem da dicotomia J), Pensamento (T), Julgamento (J) e Observação (S) - acrônimo $\mathrm{T} / \mathrm{J} / \mathrm{S}$. Apresentação envolve características como Organização (S e J), Criatividade (S e P), Intuição $(\mathrm{N})$ e Percepção $(\mathrm{P})$ - acrônimo J/N/P/S. Debate envolve características como: Consenso ( $\mathrm{F}$ e P), Percepção (P), Sentimento (F), e Pensamento $(\mathrm{T})$ - acrônimo $\mathrm{F} / \mathrm{T} / \mathrm{P}$. Criação exige características relacionadas à criatividade (combinação das dicotomias $\mathrm{S}$ e $\mathrm{P}$ ), Observação (S), Percepção (P), Intuição (N) e Sentimento (F) - acrônimo S/P/N.

Este artigo propõe o design de um artefato para sumarização colaborativa de informações de textos científicos remotamente, a partir das personalidades de integrantes de um grupo de trabalho conforme indicadores tipológicos. Desta forma, é possível apontar para um conjunto mínimo de informações desejáveis para a compreensão de um texto, permitindo que anotações sejam feitas segundo a ordenação desejada por grupos de trabalho segundo o framework FGT de Tardelli et al. (2019).

\section{Estrutura SIRColab}

Com base na metodologia DSR, foi desenvolvido um artefato no formato de formulário colaborativo $^{1}$, com o objetivo de auxiliar os pesquisadores na extração de elementos em um artigo científico em situações de trabalho remoto. Desta forma, foi realizada a instanciação da pesquisa no Mapa de DSR [Pimentel et al. 2019] (Figura 1).

A estrutura busca facilitar o levantamento de informações no estudo de um artigo considerando aspectos como tempo e personalidade dos participantes. A partir do problema de pesquisa, foram apresentadas 3 conjecturas teóricas para justificar a concepção do artefato: i) Estudantes possuem maior facilidade para explicitar seus conhecimentos tácitos através do trabalho colaborativo; ii) Personalidade é um fator que

\footnotetext{
${ }^{1}$ https://github.com/Lu-Brito/SIRColab.
} 
influencia na colaboração e iii) A utilização de uma estrutura pode facilitar a comunicação de informações sobre artigos entre os estudantes.

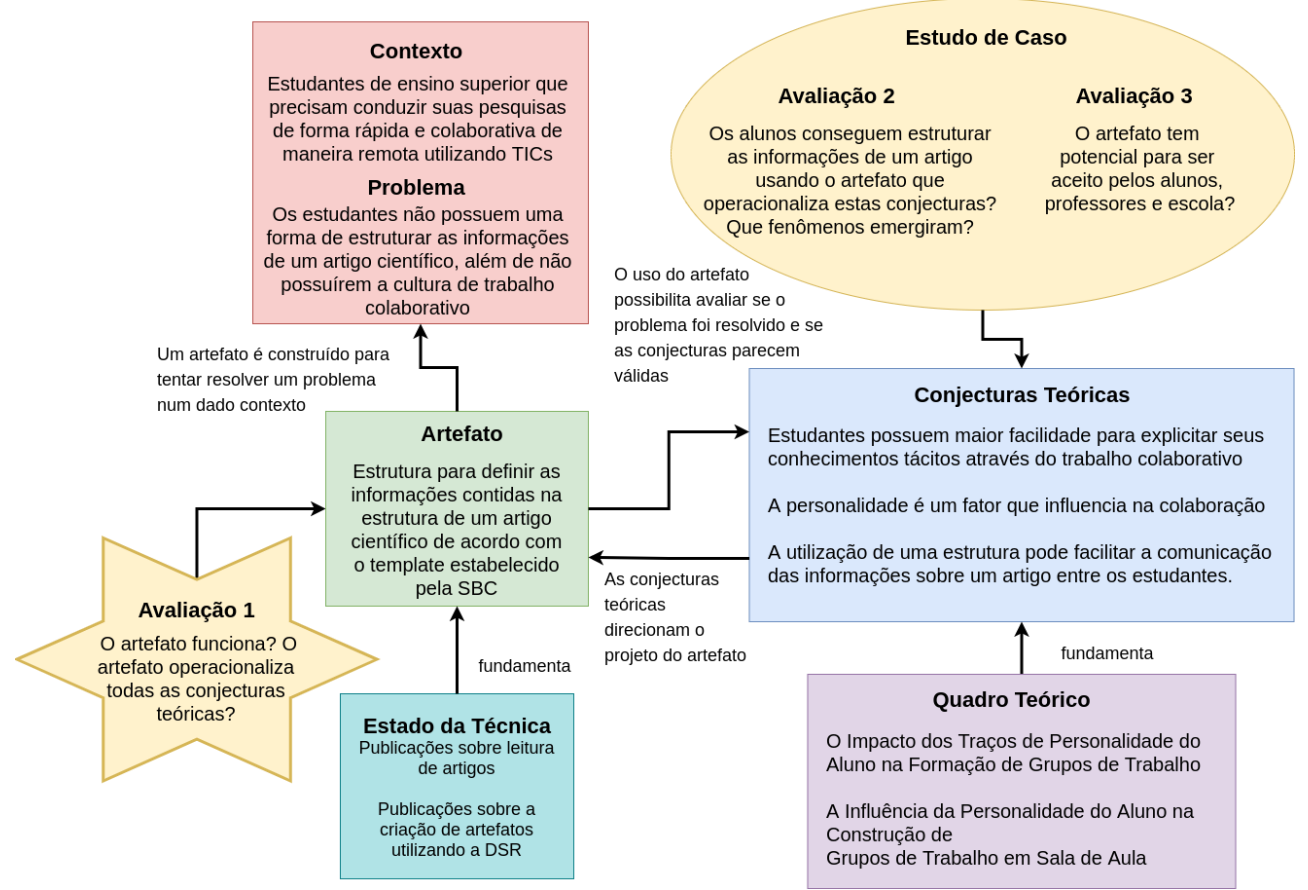

Figura1. Mapa do artefato da pesquisa, Adaptado de Pimentel et al. (2019).

O levantamento das conjecturas foi baseado em quadros teóricos que utilizam estruturas para analisar a colaboração em grupo de acordo com a personalidade dos participantes, como em Tardelli et al. (2019) e Tardelli et al. (2020). A análise do quadro teórico possibilitou comparar as diferenças das características predominantes de acordo com as dicotomias dos participantes em ambientes presenciais e remotos.

Seguindo o processo de pesquisa proposto pela abordagem DSR, foi necessária a concepção de um artefato para ajudar a resolver o problema de pesquisa. Com base na análise da publicação "Co-Creating the Workplace: Participatory Efforts to Enable Individual Work at the Hoffice [Rossitto e Lampinem 2018], foram levantados os principais elementos que compõem um artigo genérico. A escolha do artigo para análise foi realizada aleatoriamente. Foi possível inserir as categorias salientes em um template, no qual os participantes poderiam preencher os campos de maneira colaborativa ao longo de uma sessão. No artefato, 16 perguntas de pesquisa foram levantadas para preenchimento durante o processo colaborativo. Além disso, é possível definir o tipo de atividade realizada de acordo com as categorias do Framework FGT, determinando quais as dicotomias principais necessárias para cada grupo de acordo com a atividade.

A Figura 2 representa as 16 perguntas do artefato organizadas em 3 categorias de síntese de informações textuais, de acordo com o nível de aprofundamento necessário na leitura. Obteve-se as categorias a partir do uso da estrutura SIRColab em fase de testes. As perguntas da categoria em verde representam metadados descritivos que podem ser extraídos através de levantamento preliminar do artigo e através da busca de informações mais objetivas, como o ano de sua publicação e os seus conceitos-chave relacionados. Já os itens da coluna azul representam perguntas que podem ser respondidas através da leitura das seções iniciais de um artigo, detalhando seus aspectos metodológicos. Os itens 
da coluna roxa representam aspectos relacionados à descoberta de conhecimento através da pesquisa, demandando leitura mais aprofundada do texto.
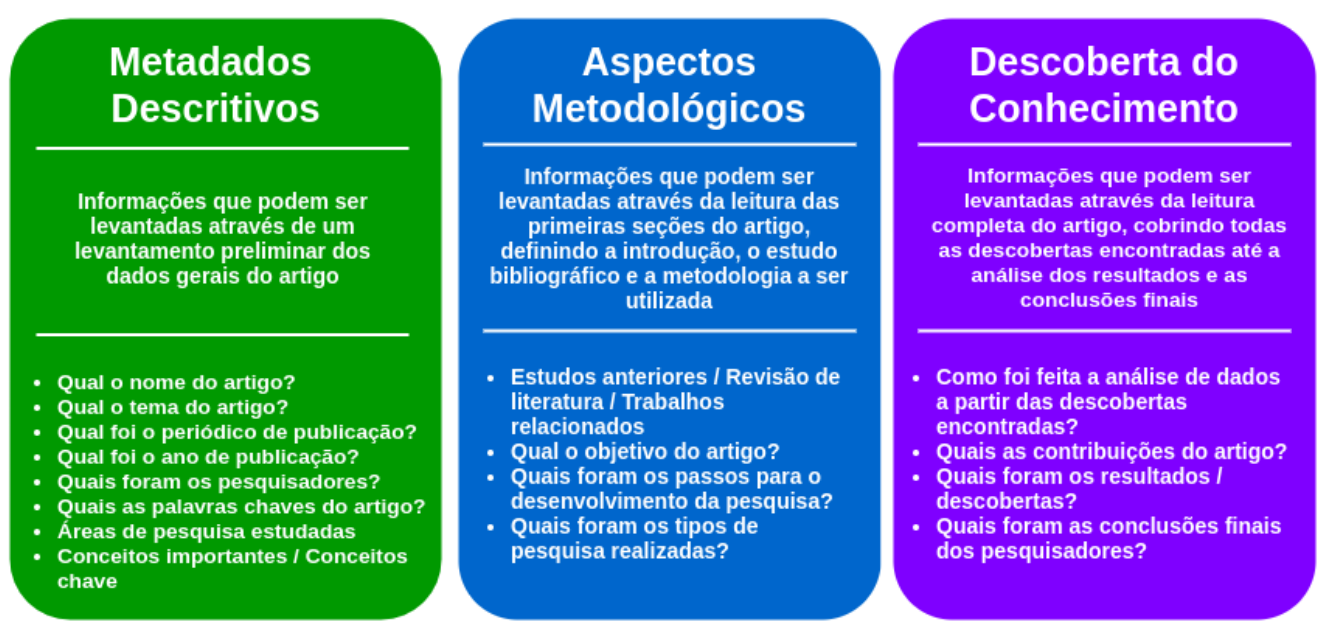

Figura 2. Categorização das perguntas levantadas na concepção do artefato.

\section{Avaliação do Artefato: Estudo de Caso}

No estudo de caso, analisou-se: configuração dos grupos, abordagens e estratégias de preenchimento do artefato e se as características e táticas dos integrantes presentes coexistiriam de maneira sinérgica, possibilitando maior engajamento na leitura colaborativa e maior eficiência no preenchimento do artefato.

\subsection{Estudo de Caso}

A avaliação do artefato foi feita através de um estudo de caso para analisar o seu uso através de uma atividade colaborativa em sala de aula remota. Após a realização do estudo, comparou-se as informações obtidas a fim de identificar eventos e padrões de ação em comum, para validação dos aspectos colaborativos e comportamentais apresentados. A Figura 3 mostra as etapas da avaliação do artefato.

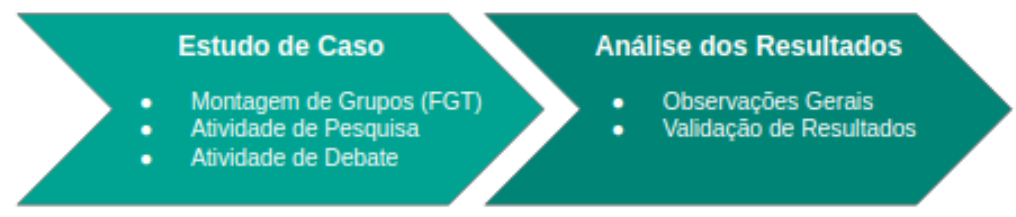

Figura 3. Esquema definindo as duas etapas do estudo exploratório.

O primeiro ciclo do estudo de caso foi realizado com uma turma de pós-graduação do curso de Ciência da Computação de uma universidade federal brasileira. Os estudantes participaram da atividade através de um aplicativo de videoconferência, concedendo autorização para o uso de suas imagens e publicação dos resultados. Com a atividade, pretendia-se verificar se a estrutura SIRColab ajuda a responder o problema de pesquisa em conjunto com as configurações geradas pelo Framework FGT.

Foram propostas 2 atividades. Pesquisa: consistia na definição da estratégia de leitura a ser abordada. Debate: consistia na discussão dos tópicos principais a serem preenchidos de acordo com as suas descobertas na estrutura SIRColab. Os integrantes 
foram agrupados de acordo com os resultados obtidos nos testes de personalidade realizados (16Personalities). O Grupo I foi configurado com dicotomias equilibradas, enquanto o Grupo II reuniu integrantes com traços Sentimentais e Julgadores (dicotomias F e J) e o Grupo III reuniu traços Observadores e Julgadores (dicotomias S e J). As dicotomias registradas podem ser observadas na Tabela 1.

Tabela 1. Dicotomias dos participantes do primeiro ciclo do estudo de caso.

\begin{tabular}{|c|l|l|l|l|}
\hline Grupo I & INFP & ENTJ & ESFJ & ISFJ \\
\hline Grupo II & ISFJ & ENFJ & ENFJ & INFJ \\
\hline Grupo III & INFJ & ESTJ & ISFJ & \\
\hline
\end{tabular}

Os integrantes de cada grupo foram organizados em salas de videoconferências Google Meet distintas, proporcionando ambientes isolados com os integrantes e o pesquisador observador de cada grupo. Forneceu-se um documento Google Docs compartilhado para os integrantes de cada grupo, contendo o formulário. Escolheu-se estas ferramentas devido ao domínio prévio e familiaridade dos participantes com elas.

$\mathrm{O}$ artigo escolhido para que os estudantes preenchessem a estrutura SIRColab durante a atividade foi "Tapetes Musicais Inteligentes" de SANTOS et al. (2016). Junto ao formulário, foi fornecido um exemplo prático já realizado com outro artigo. Os integrantes foram avisados sobre o tempo para a realização das atividades. O tempo total de atividade foi de 30 minutos ( 15 de pesquisa e 15 de debate e preenchimento).

\subsection{Estratégias e Personalidades Mapeadas}

Cada grupo utilizou diferentes estratégias para alcançar a solução do problema, dada a importância do tempo para gerar a análise completa dos artigos e preenchimento pelo grupo. Parte do tempo disponível para realizar a atividade de Pesquisa foi utilizado na definição das estratégias para possibilitar a discussão do grupo na atividade de Debate.

O Grupo I utilizou a estratégia de Leitura Diagonal (skimming) para a leitura rápida, realizando a discussão e o preenchimento da atividade com base em argumentos conjuntos, realizando o preenchimento da estrutura de maneira objetiva, conjunta e consensual. O Grupo II realizou uma leitura direcionada a partir de tópicos atribuídos para cada integrante de maneira alternada, onde o primeiro integrante era responsável por preencher o primeiro tópico, seguido pelo segundo integrante no segundo tópico e assim sucessivamente. Após iniciarem a atividade de Debate, os integrantes preencheram sincronicamente os tópicos que lhe foram atribuídos. O Grupo III optou por dividir a leitura do artigo com base na sua estrutura, através da divisão do mesmo por partes entre os participantes, fazendo com que um integrante fosse responsável pela introdução do artigo, outro pelo desenvolvimento e outro pelas conclusões gerais do artigo. A contribuição dos responsáveis por tópicos introdutórios do artigo foi menor. Nenhum dos grupos conseguiu preencher todas as categorias da estrutura. Na Tabela 2 há um resumo dos grupos, junto das estratégias e tópicos preenchidos.

Tabela 2. Estratégias utilizadas pelos grupos, seguidas de seu aproveitamento. 


\begin{tabular}{|c|c|c|c|c|}
\hline Grupo & Estratégia & $\begin{array}{c}\text { Nível de } \\
\text { detalhe }\end{array}$ & $\begin{array}{c}\text { Tópicos não } \\
\text { respondidos }\end{array}$ & Categorias com Déficit \\
\hline I & $\begin{array}{c}\text { Leitura Diagonal } \\
\text { (Skimming) }\end{array}$ & Baixo & $4 / 16$ & $\begin{array}{c}\text { Aspectos Metodológicos e } \\
\text { Descoberta de Conhecimento }\end{array}$ \\
\hline II & $\begin{array}{c}\text { Leitura integral direcionada } \\
\text { pelos tópicos propostos }\end{array}$ & Alto & $5 / 16$ & Descoberta de Conhecimento \\
\hline III & Distribuição de Seções & Médio & $6 / 16$ & Aspectos Metodológicos \\
\hline
\end{tabular}

\section{Análise dos Resultados}

Foi realizada uma análise detalhada para observar se o artefato ajudou a resolver o problema de pesquisa. As notas coletadas pelos observadores com respeito ao comportamento dos grupos foram analisadas utilizando técnicas de codificação da Teoria Fundamentada em Dados. Foi feita a triangulação de dados entre comportamento dos participantes durante a atividade, resultados e considerações levantadas no debate.

Os códigos encontrados através da Teoria Fundamentada foram agrupados em 6 categorias: Coordenação, Cooperação, Comunicação, Traços de Personalidade, Problemas Técnicos e Interação com o Ambiente Externo. Estas categorias definem aspectos da colaboração através do modelo 3C (Fuks et al., 2003), traços exclusivos da personalidade dos participantes e problemas principais em ambientes remotos. Aspectos de coordenação foram evidentes durante a definição da estratégia de leitura, e aspectos cooperativos e comunicativos foram encontrados durante a atividade de debate.

\section{Conclusão e Próximos Passos}

Esta pesquisa gerou como artefato a estrutura SIRColab, as metodologias utilizadas para sua concepção, o estudo de caso proposto e as demais inferências realizadas para validar possíveis pontos de aprimoramento. $\mathrm{O}$ artefato resolveu o problema de pesquisa, mostrando-se válido para auxiliar na leitura colaborativa de textos remotamente.

Algumas observações podem ser inferidas para validar a eficiência do artefato: (i) A definição da estratégia de leitura do artigo influencia diretamente na capacidade de discussão com os integrantes. (ii) O preenchimento dos tópicos paralelamente durante a atividade de debate pode proporcionar falta de consistência nas respostas fornecidas, devido a diferentes interpretações dos participantes. (iii) alguns tópicos demandam um tempo maior para serem respondidos do que outros, necessitando de uma avaliação de sua importância de acordo com a sua relevância na qualidade dos resultados.

Deseja-se conduzir futuramente novos experimentos para validar a eficiência do artefato, e propor a realização das atividades de Pesquisa e Debate em um ambiente unificado. Com a concepção de uma interface que direcione as necessidades de pesquisa em grupo, será possível conhecer novas formas de otimizar o ensino remoto.

\section{Referências}

AGGER, Peter; STEPHENSON, Robert S.; HASENKAM, J. Michael. (2017). How to Read Scientific Publication. In: A Practical Guide to Biomedical Research. Springer, Cham, 2017. p. 55-58. 
D'ANGELO, S., \& GERGLE, D. (2018). An Eye For Design: Gaze Visualizations for Remote Collaborative Work. Proceedings of the 2018 CHI Conference on Human Factors in Computing Systems - CHI '18. No.: 349 Pages 1-12. doi:10.1145/3173574.3173923.

FUKS, Hugo et al. Do modelo de colaboração 3c à engenharia de groupware. Simpósio Brasileiro de Sistemas Multimídia e Web-Webmidia, p. 0-8, 2003.

KRAUT, R. E., Fussell, S. R., Brennan, S. E., \& Siegel, J. (2002). Understanding effects of proximity on collaboration: Implications for technologies to support remote collaborative work. Distributed work, 137-162.

MYERS, I. B., Myers, P.B. (1995) "Gifts Differing: Understanding Personality Type”Mountain View, CA: Davies-Black Publishing, 1995.

PIMENTEL, Mariano et al. (2006) Modelo 3C de Colaboração para o desenvolvimento de Sistemas Colaborativos. Anais do III Simpósio Brasileiro de Sistemas Colaborativos, p. 58-67, 2006.

PIMENTEL, Mariano; FILIPPO, Denise; SANTORO, Flávia Maria. (2019). Design Science Research: fazendo pesquisas científicas rigorosas atreladas ao desenvolvimento de artefatos computacionais projetados para a educação. Metodologia de Pesquisa em Informática na Educação: Porto Alegre: SBC, 2019.

ROSSITTO, Chiara; LAMPINEN, Airi. Co-creating the Workplace: Participatory efforts to enable individual work at the Hoffice. Computer Supported Cooperative Work (CSCW), v. 27, n. 3-6, p. 947-982, 2018.

SANTOS, Thiago Marcondes; PIMENTEL, Mariano; FILIPPO, Denise. Tapetes Musicais Inteligentes: Computação Ubíqua para apoiar a Educação Musical. Revista Brasileira de Informática na Educação, v. 24, n. 02, p. 123, 2016.

SHAFIE, Latisha Asmaak et al. Understanding collaborative academic writing among beginner university writers in Malaysia. Studies in Literature and Language, v. 1, n. 2, p. 58-69, 2010.

SUN, Tung-Tien. Active versus passive reading: how to read scientific papers? National Science Review, v. 7, n. 9, p. 1422-1427, 2020.

TARDELLI, André; França, Juliana; Dias, Angelica; Vivacqua, Adriana; Borges, Marcos. (2019). A Influência da Personalidade do Aluno na Construção de Grupos de Trabalho em Sala de Aula. (Proceedings of the SBIE 2019).

TARDELLI, André; França, Juliana; Dias, Angelica; Vivacqua, Adriana; Borges, Marcos. (2020). O Impacto dos Traços de Personalidade do Aluno na Formação de Grupos de Trabalho. Revista Brasileira de Informática na Educação, 28, 693-722.

TOSELAND, R.W.; Rivas, R. F. (2005). An introduction to group work practice. Boston: Allyn \& Bacon. 64-91.

VAN KNIPPENBERG, D.; Schippers, M. C.; (2007). Work group diversity. Annual Review of Psychology, 58: 515-541. 